\title{
OPTIMIZATION OF THE AFLP-FINGERPRINTING METHOD FOR A MOLECULAR-GENETIC CERTIFICATION OF AGRICULTURAL MICROORGANISMS
}

\author{
V.I. Safronova, E.P. Chizhevskaya, E.E. Andronov \\ All-Russia Research and Development Institute of Agricultural Microbiology, RAAS, St. Petersburg - Pushkin 196608, Russia \\ e-mail:v.safronova@rambler.ru
}

Received May 25, 2012

S u m mary

The study involved 16 strains of the Russian collection of agricultural microorganisms (RCAM) related to the genera Bacillus, Lactobacillus, Rhizobium, and Bradyrhizobium. The aim of the work was to develop a procedure of the genotypic certification of strains with AFLP-fingerprinting (amplified fragment length polymorphism), providing detection of nucleotide polymorphisms and small rearrangements. As a result the protocol of AFLP-fingerprinting, ranging from the isolation of DNA from microbial cells and ending with computer data processing is developed. It is shown that the method of AFLP-fingerprinting has a high specificity, resolution and reproducibility of results. This method can be used to obtain individual genetic passports of microorganisms deposited in the RCAM, in order to protect the copyright of the commercial strains.

\section{Keywords: microbial collections, genotypic certification of microorganisms, AFLP-fin-gerprinting.}

Scientific work on microbial culture collections involves many research programs, one of which is identification and certification of microbial cultures necessary for a safe use of microbial resources in biotechnology (including plant growing, animal husbandry, and food industry), and the copyright protection of commercial strains. Today, such studies use the methods based on physiological and biochemical properties of bacterial strains and modern molecular genetic techniques, such as BOX, ERIC, REPPCR and AFLP-fingerprinting $(1,2)$. Different variants of fingerprinting are based on the study of bacterial genomic DNA that can reveal their individual features important for documentation in strain-specific certificates. AFLP-fingerprinting (amplified fragment length polymorphism) - one of the most promising methods for molecular genetic certification of microorganisms. This method is based on the analysis of length polymorphism of restricted and amplified DNA fragments (3, 4). High sensitivity of AFLP allows using it for identification of individual profiles of bacterial strains and distinguishing them within one species. Previously, AFLPfingerprinting was successfully applied in studies of genetic differences between closely related strains of nodule bacteria belonging to different genera (5-7).

In this regard, the purpose of this work was establishing the technique for genetic certification of bacterial strains maintained in Russian Collection of Agricultural Microorganisms based on AFLP-fingerprinting.

Technique. The objects of study were 16 practically valuable bacterial strains from Russian Collection of Agricultural Microorganisms (RCAM): Rhizobium leguminosarum (348, 700, 261, and 712), Rhizobium galegae 912, Bradyrhizobium japonicum 630, Bradyrhizobium sp. 820, Bacillus thuringiensis (626, 634, 603, 640, and 642), as well as Lactobacillus plantarum (616, 621, 631, and 613). The strains of nodule bacteria were cultured on yeast-mannitol agar (YMA) at $28{ }^{\circ} \mathrm{C}$, Bacillus thuringiensis - on meatpeptone agar (MPA), lactobacteria - on liquid wort (8).

Total DNA of nodule bacteria was isolated using the following method: $1,5 \mathrm{ml}$ overnight culture of rhizobial cells was centrifuged at $14000 \mathrm{rpm}$ for $2 \mathrm{~min}$. The supernatant was removed and the cells were resuspended in $500 \mathrm{ul} \mathrm{TE} \mathrm{buffer} \mathrm{(Tris-HCl} \mathrm{-} 10$ $\mathrm{mmol} / \mathrm{l}$, EDTA - $5 \mathrm{mmol} / \mathrm{l}, \mathrm{pH} 8,0)$. The suspension was added with lysozyme $(1 \mathrm{mg} / \mathrm{ml})$, incubated for 5 min at room temperature, then introduced SDS up to the concentration $0,5 \%$ and proteinase $\mathrm{K}$ to the concentration $0,05 \mathrm{mg} / \mathrm{ml}$, and incubated at $37{ }^{\circ} \mathrm{C}$ for $1 \mathrm{~h}$.

Extraction mixture - phenol, chloroform and isoamyl alcohol (24:24:1). DNA was precipitated with two volumes of ethanol for $5 \mathrm{~min}$ at room temperature. The solution was centrifuged at $14000 \mathrm{rpm}$ for $2 \mathrm{~min}$ and the precipitate was dissolved in $100 \mathrm{ul}$ distilled water. In the case of Bacillus and Lactobacillus, the extraction of DNA included pretreatment with proteinase $\mathrm{K}$ at a concentration of $0,05 \mathrm{mg} / \mathrm{ml}$ at $60{ }^{\circ} \mathrm{C}$ for $1 \mathrm{~h}$; if necessary, cell disruption was provided by shaking the suspension with glass beads in a homogenizer FastPrep24 ("MP Biomedicals", USA) at maximum power for $1 \mathrm{~min}$. Final purification was carried out as described above.

The resulting genomic DNA (50 ng) was used for simultaneous restriction and ligation reactions. Composition of the reaction mixture: restriction enzymes EcoRI and MseI - 2,5 units of each ("Fermentas", Lithuania), 1 unit of T4 phage ligase ("Fermentas", Lithuania), two oligonucleotide adapters - for the site EcoRI (adEco1 CTCGTAGACTGCGTACC and adEco2 AATTGGTACGCAGTCTAC) and for the site MseI (adMse1 GACGAGAGTCCTG-AG and adMse2 TACTCAGGACTCAT) at 5 pmol of each. The reaction was carried out in ligase buffer ("Fermentas", Lithuania) at $37^{\circ} \mathrm{C}$ for $16 \mathrm{~h}$.

Genomic fingerprints were obtained after the final amplification using as template $1 \mathrm{ul}$ of the reaction mixture obtained in the previous stage, and selective primers (one for each adapter labeled with fluorescent dye FAM): Mse_a GA-TGAGTCCTGAGTAAA, Mse_cg GATGAGTCCTGAGTAACG or Mse_ca GATGAGTCCTGAGTAACA - for the site MseI; Eco_0 GACTGCGTACCAATT or Eco_a GACTGCGTACCAATTCA - for the site EcoRI (10 pmol of each). Searching for the optimum combination there were tested all possible combinations of MseI / EcoRI selective primers. Preliminary assessment of results was performed in 3\% agarose gel, final evaluation - by automated capillary electrophoresis in genetic analyzer ABI 3500xl (“Applied Biosystems", USA). The internal molecular weight marker GeneScan-600 LIZ Size Standard ("Applied Biosystems", USA) was also added to the mix for electrophoretic separation.

Comparison of the fingerprints was performed in FPQuest program ("Bio Rad", USA). The source material for the analysis were the files resulting from electrophoretic separation of the fragments and containing the curves corresponding to AFLP fragments (FAM) and molecular weight standard (LIZ). For this purpose, the files were pre-converted to TIFF-format.

The degree of similarity of the fingerprints was assessed by Pearson's correlation coefficient, which is known to be focused rather on detection of common similarities of curves than fragment size. 


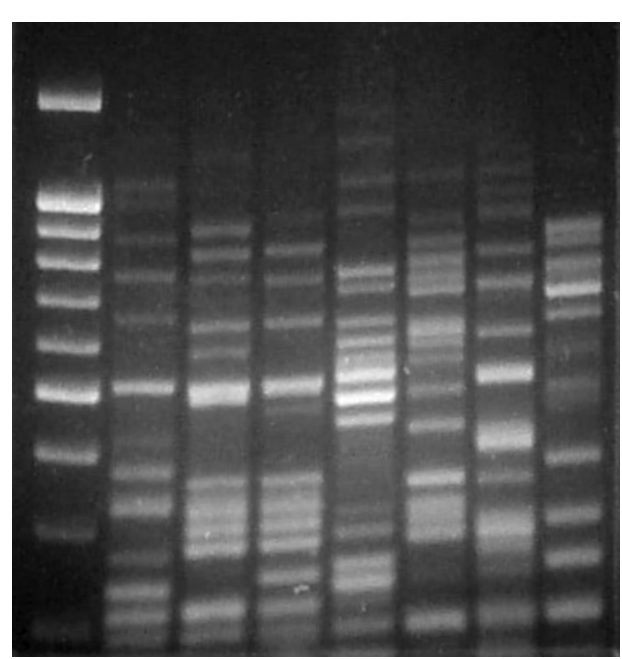

Fig. 1. Pre-electrophoresis in $3 \%$ agarose gel of AFLP-fragments of rhizobial DNA derived with the use of selective primers Mse_ca/Eco_a.
Results. Genomic DNA was isolated from all analyzed strains, and its concentration and degree of purity satisfied the conditions of AFLP. Restriction and ligation with adapters provided excellent results - using restriction-ligation mixture as a template in the final stage of AFLP-PCR resulted in highly specific profiles for all tested microorganisms. After the study of different combinations of selective primers, the pair Mse_ca/Eco_was selected as it gave an optimal ratio of the amount and specificity of resulting fragments (Fig. 1).

Processing of the obtained data in FPQuest program allowed effective alignment of the fingerprints, calculation of their relationship and clustering based on statistical support. Firstly, the dendrogram of relationship was constructed using all the obtained data, including different strains and different volumes of the mixture of fragments used in electrophoresis $(1 ; 0,5 ; 0,25 ; 0,11)$ in order to select the optimum ratio for effective separation and elimination of effects associated with overload or insufficient amount of the introduced material (Fig. 2).

Analysis of the data showed that, except the strains with high level of similarity (Lactobacillus 621, 616, 631, and 613; Bacillus thuringiensis 626/634 and 640/642), the fingerprints were grouped exactly by strains with very high similarity of profiles corresponding to different amounts of AFLP-mix and belonging to the same strain. From the results of comparison it was found the optimal amount of AFLP-mix for application in genetic analyzer equal to $0,1 \mathrm{ul}$. Therefore, the samples with the lowest concentration were used in final comparison (Fig. 3). The analysis of these samples revealed clear differences in sets of DNA fragments indicating a very high sensitivity of the method allowing to obtain unique profiles for each strain and discriminate them within a species. At the same time, even with such high specificity of this method, cluster analysis of AFLP-profiles allows exact grouping of bacterial strains in terms of their phylogenetic relationship. The dendrogram (Fig. 3) shows the strains of rhizobia (348, 912, 630, 820, 700, 261, and 712), Bacillus (626, 634, 603, 640, and 642), and Lactobacillus (616, 621, 631, and 613) as separate groups. Among the analyzed nodule bacteria, five strains - 348 (Rhizobium leguminosarum bv. trifolii), 912 (Rhizobium galegae), 630 (Bradyrhizobium japonicum), 820 (Bradyrhizobium sp.), 700 (Rhizobium leguminosarum bv. Phaseoli) appeared to be outside the clusters (degree of similarity less than $30 \%$ ). 


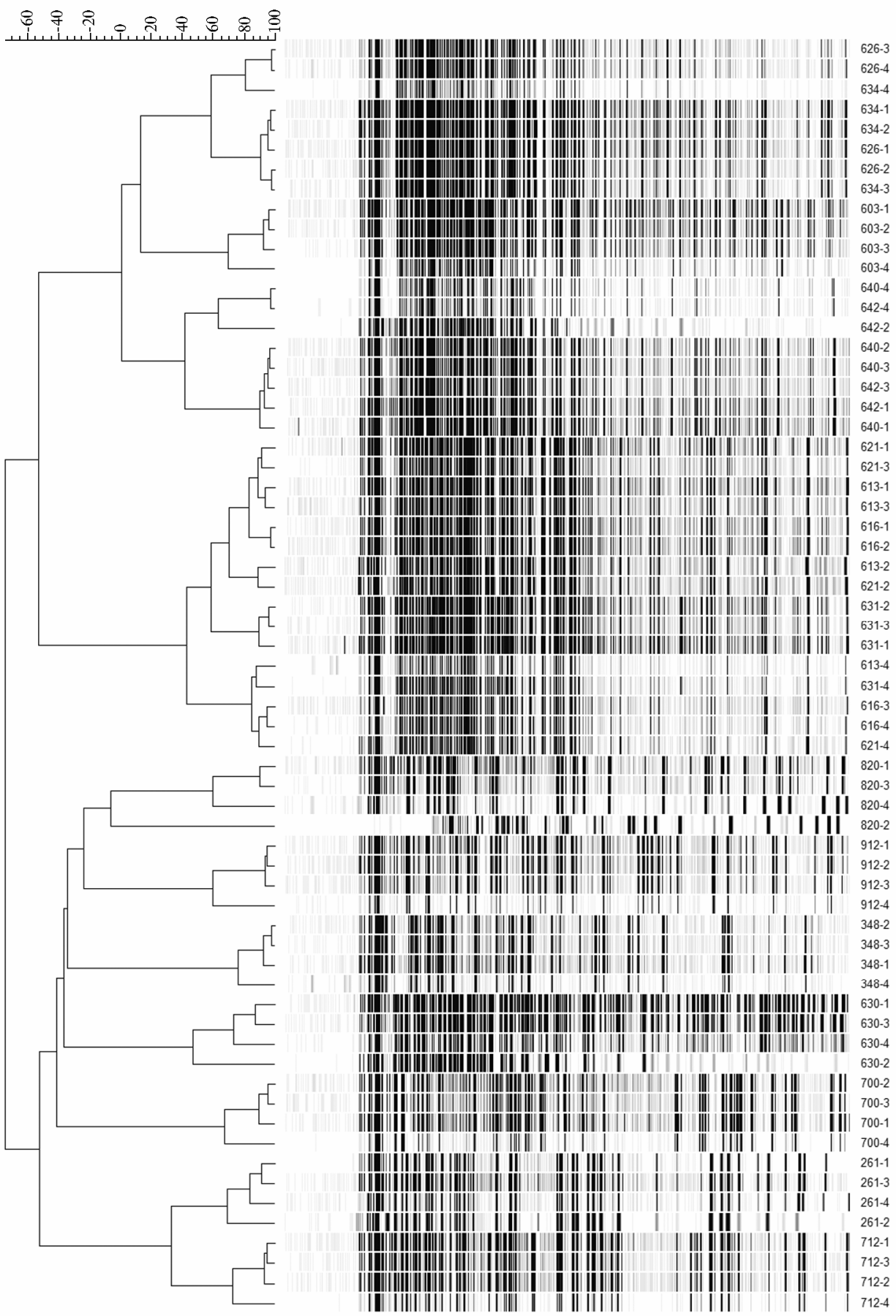

Fig. 2. Comparison of total data (all bacterial strains and all quantities) obtained at separation of AFLPfragments on genetic analyzer (ABI 3500xl, "Applied Biosystems", USA): $1-1 \mathrm{ul}, 2-0,5 \mathrm{ul}, 3-0,25 \mathrm{ul}, 4$ $-0,1 \mathrm{ul}$.

The other two strains of rhizobia (261 and 712 Rhizobium leguminosarum bv. viciae), as well as the strains of Bacillus and Lactobacillus, can be considered as separate groups since the degree of their similarity $50 \%$ was sufficient for statistically reliable isolation of clusters $(9,10)$. 

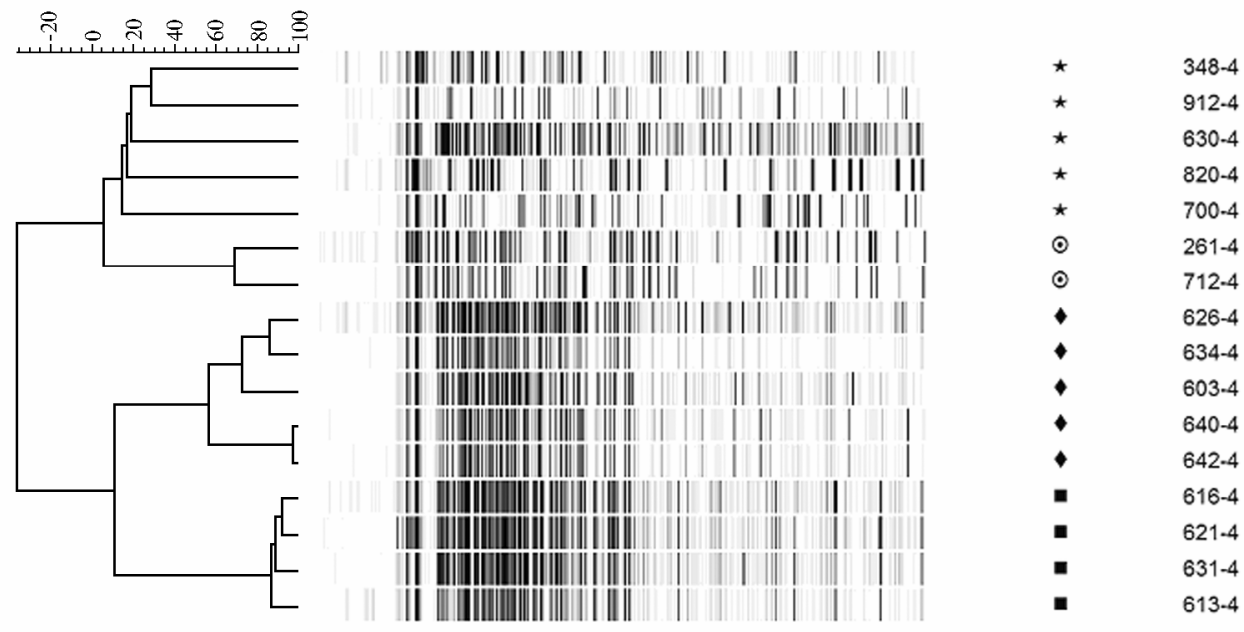

Fig. 3. Comparison of data of AFLP-fingerprinting in all analyzed strains of agricultural microorganisms corresponding to the introduction of 0,1 ul AFLP-mix.

Thus, AFLP-fingerprinting has demonstrated its high accuracy in differentiation of genotypes at both intra- and interspecific level. This highly reproducible method has a number of effective approaches for analysis of resulting data, and it can be successfully used in crop production, animal husbandry, and food industry for molecular genetic characterization of economically important strains of microorganisms. Results of this work will be used in establishing the standard procedure of a comprehensive evidence-based certification of strains of agricultural microorganisms deposited in Russian Collection of Agricultural Microorganisms (RCAM).

\section{REFERENCES}

1. Vinuesa P., Rademaker J.L.W., De Bruijn F.J., and Werner D., Genotypic Characterization of Bradyrhizobium Strains Nodulating Endemic Woody Legumes of the Canary Islands by PCR-Restriction Fragment Length Polymorphism Analysis of Genes Encoding 16S rRNA (16S rDNA) and 16S-23S rDNA Intergenic Spacers, Repetitive Extragenic Palindromic PCR Genomic Fingerprinting and Partial 16S rDNA Sequencing, Appl. Environ. Microbiol., 1998, vol. 64, pp. 20962104.

2. Vos P., Hogers R., Bleeker M., et al., AFLP: a New Technique for DNA Fingerprinting, Nucl. Acids Res., 1995, vol. 23 , pp. $4407-4414$.

3. Willems A., Doignon-Bourcier F., Coopman R., et al., AFLP Fingerprint Analysis of Bradyrhizobium Strains Isolated from Faidherbia albida and Aeschynomene Species, System. Appl. Microbiol., 2000, vol. 23, pp. 137-147.

4. Paun O. and Schönswetter P., Amplified Fragment Length Polymorphism: an Invaluable Fingerprinting Technique for Genomic, Transcriptomic, and Epigenetic Studies, Methods Mol. Biol., 2012, vol. 862, pp. 75-87.

5. Wdowiak-Wróbel S. and Małek W., Genomic Diversity of Astragalus cicer Microsymbionts Revealed by AFLP Fingerprinting, J. Gen. Appl. Microbiol., 2005, vol. 51 , pp. 369-378.

6. Safronova V., Chizhevskaya E., Bullitta S., Andronov E., Belimov A., Charles T.C., and Lindström K., Presence of a Bovel 16S-23S rRNA Gene Intergenic Spacer Insert in Bradyrhizobium canariense Strains, FEMS Microbiol. Lett., 2007, vol. 269, pp. 207-212.

7. Aserse A.A., Räsänen L.A., Assefa F., Hailemariam A., and Lindström K., Phylogeny and Genetic Diversity of Native Rhizobia Nodulating Common Bean (Phaseolus vulgaris L.) in Ethiopia, Syst. Appl. Microbiol., 2012, vol. 35, pp. 120-131.

8. Safronova V.I., Osledkin Yu.S., Sviridova O.V., and Vorobyov N.I., Metody konservatsii kollektsionnykh kul'tur mikroorganizmov: Metod. rekom. (Guidelines on Methods for the Conservation of Collections of Microbial Cultures), St. Petersburg, 2007.

9. Janssen P., Coopman R., Huys G., et al., Evaluation of the DNA Fingerprinting Method AFLP as a New Tool in Bacterial Taxonomy, Microbiology, 1996, vol. 142, pp. 1881-1893.

10. Willems A., Doignon-Bourcier F., Coopman R., et al., AFLP Fingerprint Analysis of Bradyrhizobium Strains Isolated from Faidherbia albida and Aeschynomene Species, System. Appl. Microbiol., 2000, vol. 23, pp. 137-147. 